\title{
Initial Management of Severe Hyperglycemia in Patients with Type 2 Diabetes: an Observational Study
}

William M. King IV • Michael T. McDermott •

Jennifer M. Trujillo

To view enhanced content go to www.diabetestherapy-open.com

Received: June 19, 2013 / Published online: August 16, 2013

(C) The Author(s) 2013. This article is published with open access at Springerlink.com

\section{ABSTRACT}

Introduction: Current guidelines recommend insulin for patients with type 2 diabetes (T2D) and severe hyperglycemia, but this recommendation lacks sufficient evidence and poses practical challenges. It is unclear whether non-insulin treatments are effective in this setting. The objective of this study was to describe treatment strategies of T2D patients with severe hyperglycemia and identify which initial treatments, interventions, or patient characteristics correlated with successful glucose lowering.

During the time this project was conducted, Dr. King was a PGY1 Pharmacy Resident at the University of Colorado Hospital.

W. M. King IV · J. M. Trujillo ( $\square)$

University of Colorado Skaggs School of Pharmacy and Pharmaceutical Sciences, Aurora, CO, USA e-mail: jennifer.trujillo@ucdenver.edu

M. T. McDermott

University of Colorado Hospital, Aurora, CO, USA

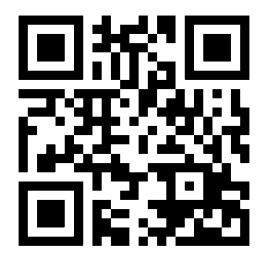

Enhanced content for this article is

available on the journal web site:

www.diabetestherapy-open.com
Methods: This was a retrospective cohort study of 114 patients with T2D and a glycosylated hemoglobin (A1C) $\geq 12 \%$. Changes in $\mathrm{A} 1 \mathrm{C}$ were compared between patients started on non-insulin medications versus insulin-based regimens. Regression analysis was performed to assess predictors of success in achieving A1C $\leq 9 \%$ within 1 year. The main outcomes measures were change in $\mathrm{A} 1 \mathrm{C}$ from baseline and predictors of success in achieving $\mathrm{A} 1 \mathrm{C} \leq 9 \%$ within 1 year.

Results: At baseline, 43 patients (37.7\%) started one or more non-insulin medications; 71 (62.3\%) started insulin. Fifty-eight patients (50.8\%) achieved an A1C $\leq 9 \%$. Predictors of success were newly diagnosed T2D, certified diabetes educator (CDE) visits, and less time to follow-up A1C; insulin therapy was not. Change in A1C was significantly better in the non-insulin cohort compared to the insulin cohort $(-4.5 \%$ vs. $-2.8 \%, p=0.001)$. Newly diagnosed patients were less likely to start insulin therapy $(20.8 \%$ vs. $73.3 \%, p<0.001)$, less likely to use insulin at any point ( $29.2 \%$ vs $81.1 \%, p<0.001)$, and more likely to achieve an $\mathrm{A} 1 \mathrm{C} \leq 9 \%$ compared to patients with established T2D (87.5\% vs $41.1 \%$, $p<0.001)$.

Conclusion: Insulin therapy was used in roughly two-thirds of patients with severe 
hyperglycemia, but did not result in better glycemic control compared to non-insulin regimens. Rapid follow-up, more CDE visits, and a new diabetes diagnosis were predictors of successful glucose lowering. Patients with T2D and severe hyperglycemia, particularly those newly diagnosed, may be managed with noninsulin therapy.

Keywords: Diabetes mellitus type 2; Hyperglycemia; Insulin; Primary care

\section{INTRODUCTION}

Diabetes affects 25.8 million people (8.3\%) in the USA. There were 1.9 million new cases diagnosed in 2010 alone [1]. While the diagnostic threshold for diabetes is an A1C $\geq 6.5 \%$, patients can present with severe hyperglycemia with A1C values reaching higher than $14 \%$. Severe hyperglycemia may warrant specific and immediate treatment considerations to prevent acute complications, alleviate symptoms, and preserve beta cell function $[2,3]$. However, literature regarding the effectiveness of different treatment approaches to manage patients with non-emergent, severe hyperglycemia is limited.

The American Diabetes Association's (ADA) position statement on a patient-centered approach to the management of hyperglycemia in type 2 diabetes recommends that "if a patient presents with significant hyperglycemic symptoms and/or has dramatically elevated plasma glucose concentrations ( $>300-350 \mathrm{mg} /$ $\mathrm{dL}$ ), or an $\mathrm{A} 1 \mathrm{C} \geq 10-12 \%$, insulin therapy should be strongly considered from the outset" [3]. After symptoms are relieved and glucose levels are decreased, oral agents can be added and it may be possible to withdraw insulin, if preferred. This recommendation makes no distinction between newly diagnosed or drug-naïve patients and treated or established patients, and does not provide references or level of evidence.

The American Association of Clinical Endocrinologists (AACE) algorithm for glycemic control recommends insulin therapy \pm other agents for patients who present with an A1C >9\% [4]. The recommendations state that "for drug naïve patients with A1C levels $>9 \%$, it is unlikely that 1,2 , or even 3 agents (other than insulin) will achieve the A1C goal". The algorithm does, however, suggest that treatment with combination non-insulin agents in drug-naïve patients who do not have symptoms may be sufficient. References or levels of evidence are not provided.

Although consensus statements suggest initiating insulin therapy, this is challenging in real-world practice. Successful insulin initiation requires time, resources, knowledge, proper dose titration, significant patient education and training, self-monitoring of blood glucose, and frequent follow-up [3]. In the primary care setting, many barriers exist, including time restraints, patient resistance, and lack of available resources and trained staff that make it difficult to initiate insulin therapy in a safe and effective manner.

The lack of sufficient evidence and the practical challenges posed by initiating insulin therapy support the need for further evaluation of treatment approaches to severe hyperglycemia in T2D patients. Therefore, the purpose of this study was to (1) describe the patient characteristics and initial management strategies of patients with T2D who presented with $\mathrm{A} 1 \mathrm{C}$ values $\geq 12 \%$, (2) compare the changes in $\mathrm{A} 1 \mathrm{C}$ from baseline to 12 months between patients prescribed insulin-based treatment versus patients prescribed non-insulin-based treatment, and (3) identify which initial treatments, interventions, or patient 
characteristics correlated with clinically meaningful glycemic response.

\section{MATERIALS AND METHODS}

\section{Study Design}

This study was a retrospective observational cohort study of patients with a new or established diagnosis of type 2 diabetes presenting within the University of Colorado Hospital (UCH) ambulatory clinic system with non-emergent, severe hyperglycemia, defined as a measured hemoglobin $\mathrm{A} 1 \mathrm{C}$ of $\geq 12 \%$. Clinics were primarily internal medicine and family medicine practices. The study was approved by the Colorado Multiple Institutional Review Board and the Hospital Research Review Board for the UCH. Patients with an A1C value $\geq 12 \%$ during the time period of January 1, 2006 to March 31, 2011 were identified. Electronic medical records (EMR) for each patient were reviewed. Data were reviewed from the date of initial presentation of patients with $\mathrm{A} 1 \mathrm{C} \geq 12 \%$ (defined as baseline) and followed through 1 year. Study data were collected and managed using a secure, electronic data capture tool [5]. Data collection included patient demographics (age, sex, weight, race/ethnicity, duration of diabetes, insurance status), initial treatment for diabetes within 1 month of initial presentation, diabetes medications subsequently used after 1 month, A1C values from date of initial presentation through 1 year, the number of visits with an endocrine provider or a certified diabetes educator, the number of total clinic visits with diabetes listed in the medical problem list, the number of hospitalizations or emergency department visits with diabetes listed in the medical problem list, and time to first follow-up visit after initial A1C level $\geq 12 \%$.
The analysis in this article is based on previously conducted studies and does not involve any new studies of human or animal subjects performed by any of the authors.

\section{Patient Population}

Patients were included in the study if they were at least 18 years of age, had an $\mathrm{A} 1 \mathrm{C} \geq 12 \%$ drawn from a UCH ambulatory clinic, and had a new or established diagnosis of type 2 diabetes. Patients were excluded if they were less than 18 years of age, had type 1 diabetes, had no follow-up A1C reading within 12 months of initial presentation, were pregnant, were taking corticosteroids, or were diagnosed with stress-induced hyperglycemia, hyperglycemic hyperosmolar state, or diabetic ketoacidosis.

\section{Study Cohorts}

\section{Clinical Response Cohorts}

Patients were categorized into two cohorts based on a clinically meaningful improvement in glycemic control: (1) responders, defined as those patients who achieved an A1C $\leq 9 \%$ within 1 year of baseline, and (2) nonresponders, those who did not achieve an A1C $\leq 9 \%$ within 1 year of baseline. A regression analysis was performed to identify what factors served as predictors of success in achieving an $\mathrm{A} 1 \mathrm{C} \leq 9 \%$.

\section{Initial Treatment Cohorts}

Patients were stratified into two cohorts based on initial treatment medication prescribed within 1 month of baseline: (1) one or more non-insulin medications and (2) one or more insulin medications with or without noninsulin medications. Non-insulin medications included metformin, glyburide, glipizide, 
glimepiride, sitagliptin, saxagliptin, linagliptin, pioglitazone, rosiglitazone, repaglinide, nateglinide, acarbose, miglitol, pramlintide, liraglutide, or exenatide. Changes in A1C from baseline to 12 months were compared between the cohorts using a last observation carried forward method.

\section{Outcomes}

The outcomes of the study were: (1) descriptive results of patient characteristics and initial management strategies of patients with T2D who presented with A1C values $\geq 12 \%$, (2) predictors of success that correlated with clinically meaningful glycemic response, defined as achieving an $\mathrm{A} 1 \mathrm{C} \leq 9 \%$ within 1 year, and (3) change in A1C from baseline to 12 months between patients prescribed insulinbased treatment versus patients prescribed noninsulin-based treatment.

\section{Statistical Analysis}

Demographic measures were summarized by their mean (SD) or $n$ (\%) for categorical measures. Comparisons of mean and categorical frequencies across the clinical response cohorts were tested using the $t$ test or the Chi-squared test. The Welch corrected $t$ test was used for continuous data that were not normally distributed. Comparison of baseline A1C to the end-point $\mathrm{A} 1 \mathrm{C}$ was conducted using paired $t$ test. Comparing changes in $\mathrm{A} 1 \mathrm{C}$ between the cohorts was conducted using two sample $t$ tests and the analysis of covariance (ANCOVA) model using baseline A1C and diagnosis status as covariates. These analyses were conducted using last observation carried forward. Logistic regression analysis was used to determine predictors of clinical response. Days to first follow-up, days to first A1C follow-up, number of endocrine visits, number of CDE appointments, number of other visits, insulin medication prescribed at any point during the year, and health insurance status (public or none vs. private) were considered as explanatory variables. The a priori level of significance was defined as $p<0.05$. All analyses were performed using SAS 9.3 software (SAS Institute, Inc., Cary, NC, USA).

\section{RESULTS}

A total of 242 patients were identified who presented to a UCH outpatient clinic with an A1C $\geq 12 \%$ during the study period. After excluding patients primarily due to type 1 diabetes diagnosis or lack of follow-up A1C, a sample of 114 patients was included in the final analysis. The average age of the patient population was 53 years, $64(47.4 \%)$ were male, and $84(73.7 \%)$ were non-Hispanic. The average baseline A1C was $13.1 \%$, average weight was $91.9 \mathrm{~kg}$, and average duration of diabetes was 10 years. Twenty-four patients (21.1\%) did not have a previous diagnosis of T2D at the time of presenting with an $\mathrm{A} 1 \mathrm{C} \geq 12 \%$. Initial treatment strategies are shown in Table 1. Within 1 month of baseline, 43 patients (37.7\%) started one or more non-insulin medications rather than initiating insulin. Of those, 20 started a single non-insulin medication, the majority of which was metformin. Seventy-one patients (62.3\%) initially started an insulin-based regimen.

Of the 114 patients, 58 (50.8\%) were classified as responders (i.e., achieved an A1C $\leq 9 \%$ within 1 year) and $56(49.1 \%)$ were classified as non-responders. Comparisons between responders and non-responders are in Table 2. Responders were slightly older ( 54.7 vs 50.7 years, $p=0.032$ ) and more likely to be newly diagnosed with T2D compared to non-responders $(36.2 \%$ vs $5.4 \%, p<0.001)$. 
Table 1 Initial management strategies

\begin{tabular}{ll}
\hline $\begin{array}{l}\text { Initial management strategy } \\
\text { within } \mathbf{1} \text { month of baseline }\end{array}$ & $\begin{array}{l}\text { Number of } \\
\text { patients (\%) }\end{array}$ \\
\hline One or more non-insulin medications & $43(37.7)$ \\
Single non-insulin medication & $20(17.5)$ \\
Two or more non-insulin medications & $23(20.2)$ \\
Insulin-based regimen & $71(62.3)$ \\
Insulin plus non-insulin & $43(37.7)$ \\
Basal insulin alone & $10(8.8)$ \\
Basal plus bolus insulin & $18(15.8)$ \\
\hline
\end{tabular}

Responders were less likely to initiate therapy with insulin $(51.7 \%$ vs $73.2 \%, p=0.019)$ and more likely to be initiated on a single non-insulin medication $(25.9 \%$ vs $8.9 \%$, $p=0.014)$. Responders were found to have significantly more visits with a CDE $(1.2 \pm 1.5$ vs $0.4 \pm 1, p=0.002$ ) and more likely to have fewer days to the first follow-up A1C (120 \pm 72.6 vs. $154.7 \pm 97.5, p=0.033)$. Significantly more patients received insulin at some point in the year in the non-responder group compared to the responder group $(82.1 \%$ vs $58.6 \%$, $p=0.006)$.

Results of the regression analysis to determine predictors of response are in Table 3. A new diagnosis of diabetes, less days to first $\mathrm{A} 1 \mathrm{C}$ follow-up, and more $\mathrm{CDE}$ visits were found to be predictors of response, while insulin treatment and number of endocrine clinic visits were not.

Changes in A1C from baseline are in Table 4. The average $\mathrm{A} 1 \mathrm{C}$ reduction in the total patient population was $3.5 \%$. The non-insulin-treated cohort had a significantly greater reduction in A1C compared to the insulin-treated cohort $(-4.5 \%$ vs $-2.8 \%, p=0.0011)$. Additionally, a significantly greater percentage of patients in the non-insulin cohort achieved an A1C $\leq 9 \%$ compared to the insulin-treated cohort $(65.1 \%$ vs. $42.3 \%, p=0.0179$ ). Within diagnosis status subgroups (newly diagnosed vs. established diabetes), the changes in $\mathrm{A} 1 \mathrm{C}$ from baseline were not significantly different between insulintreated and non-insulin-treated cohorts (Table 4). In addition, the ANCOVA model showed no evidence that A1C decreases differed significantly between insulin and noninsulin use after controlling for baseline A1C and diagnosis status.

A new diagnosis of T2D at the time of presenting with an $\mathrm{A} 1 \mathrm{C} \geq 12 \%$ was found to be a confounding variable in the regression analysis. Therefore, additional post hoc analyses were performed to evaluate results between newly diagnosed and previously diagnosed patients. There were significantly greater reductions in A1C in newly diagnosed patients compared to patients with established diabetes ( $-6.3 \%$ vs. $-2.7 \%, p=0.001$, Table 5$)$, with A1C differences emerging within 3-5 months from baseline (Fig. 1). Twenty-one out of 24 newly diagnosed patients (87.5\%) were classified as responders, achieving an A1C $\leq 9 \%$, which was significantly higher than the $41.1 \%$ of patients with established diabetes $(p<0.001)$. In addition, $17(70.9 \%)$ of the newly diagnosed population achieved the ADA-recommended goal A1C of $\leq 7 \%$ as opposed to 12 (13.3\%) of previously diagnosed patients. This occurred despite the fact that newly diagnosed patients were less likely to be initially treated with insulin $(20.8 \%$ vs. $73.3 \%$, $p<0.001$ ) or receive insulin at any point throughout the year $(29.2 \%$ vs. $81.1 \%$, $p<0.001)$. Of note, 16 of the 24 patients $(67 \%)$ started a single non-insulin medication; 14 of those achieved an $\mathrm{A} 1 \mathrm{C} \leq 9 \%$ and 10 of those achieved the ADA-recommended A1C of $\leq 7 \%$ within a year. Details regarding the other initial treatment regimens of newly diagnosed patients are shown in Table 6. 
Table 2 Comparisons between responders and non-responders

\begin{tabular}{lccc}
\hline Parameter & $\begin{array}{l}\text { Responders } \\
(\boldsymbol{n}=\mathbf{5 8})\end{array}$ & $\begin{array}{l}\text { Non-responders } \\
(\boldsymbol{n}=\mathbf{5 6})\end{array}$ & $\boldsymbol{p}$ value \\
\hline Mean age, years (range) & $54.7(22-74)$ & $50.7(28-72)$ & 0.032 \\
Sex, No. (\% men) & $27(46.6)$ & $27(48.2)$ & 0.86 \\
Non-hispanic, no. (\%) & $43(74.1)$ & $41(73.2)$ & 0.91 \\
Mean weight at baseline, kg (SD) & $91.9(32.6)$ & $93.2(29.0)$ & 0.82 \\
Insurance (non-government), no. (\%) & $28(48.3)$ & $27(48.2)$ & 0.99 \\
Mean baseline A1C, \% (SD) & $13.2(0.8)$ & $13.0(0.9)$ & 0.28 \\
Mean duration of diabetes, years (SD) & $10.0(7.4)$ & $10.6(7.0)$ & 0.72 \\
New T2D diagnosis at baseline, no. (\%) & $21(36.2)$ & $3(5.4)$ & $<0.001$ \\
Initial treatment with insulin-based regimen, no. (\%) & $30(51.7)$ & $41(73.2)$ & 0.019 \\
Insulin prescribed at any point in year, no. (\%) & $34(58.6)$ & $46(82.1)$ & 0.006 \\
First follow-up visit, days (SD) & $42.7(57.8)$ & $65.0(84.9)$ & 0.11 \\
First follow-up A1C, days (SD) & $120.0(72.6)$ & $154.7(97.5)$ & 0.033 \\
CDE visits, no. (SD) & $1.2(1.5)$ & $0.4(1.0)$ & 0.002 \\
Endocrine visits, no. (SD) & $1.2(1.8)$ & $1.1(1.6)$ & 0.67 \\
Other appointments, no. (SD) & $4.2(3.0)$ & $3.8(3.1)$ & 0.38 \\
\hline
\end{tabular}

$A 1 C$ glycosylated hemoglobin, $C D E$ certified diabetes educator, $T 2 D$ type 2 diabetes

Table 3 Logistic regression modeling the odds of success at any point in the year

\begin{tabular}{lcll}
\hline Parameter & $\begin{array}{c}\text { Odds } \\
\text { ratio }\end{array}$ & $\begin{array}{l}\text { Confidence } \\
\text { interval }\end{array}$ & $\boldsymbol{p}$ value \\
\hline Newly diagnosed & 11.2 & $2.3-55.6$ & 0.003 \\
$\begin{array}{l}\text { Days to first follow-up } \\
\text { A1C }\end{array}$ & 1.0 & $0.9-1.0$ & 0.042 \\
CDE visits & 1.9 & $1.2-2.9$ & 0.007 \\
Endocrine visits & 0.8 & $0.6-1.1$ & 0.21 \\
Insulin therapy & 0.1 & $0.0-0.4$ & 0.25 \\
\hline
\end{tabular}

$A 1 C$ glycosylated hemoglobin, $C D E$ certified diabetes educator

\section{DISCUSSION}

This study revealed that, although clinical guidelines recommend insulin therapy for patients with T2D presenting with severe hyperglycemia, only about two-thirds of patients in this study were initiated on an insulin-based regimen. Over one-third of patients were treated with non-insulin medications with $17.5 \%$ starting on a single non-insulin medication. Regardless of adherence to guideline recommendations, $50.8 \%$ of the total population $(n=58)$ achieved an $\mathrm{A} 1 \mathrm{C} \leq 9 \%$ by the end of the year. No correlation was found between any specific medication regimen and meaningful glucose response.

The current study provides useful insight into real-world use of insulin therapy in this patient population. Overall, insulin use in the responder group was lower than anticipated with only about half initiating insulin-based 
Table 4 Changes in A1C from baseline

\begin{tabular}{lcccc}
\hline & No. & $\begin{array}{l}\text { Baseline } \\
\text { A1C, \% (SD) }\end{array}$ & $\begin{array}{l}\text { Last A1C, } \\
\%(S D)^{\mathbf{a}}\end{array}$ & $\begin{array}{l}\text { Change in A1C } \\
\text { from baseline, \% (SD) }\end{array}$ \\
\hline $\begin{array}{l}\text { All patients } \\
\text { Treatment cohort }\end{array}$ & 114 & $13.1(0.8)$ & $9.6(2.5)$ & $-3.5(2.7)$ \\
Non-insulin treated & 43 & $13.2(1.0)$ & $8.6(2.3)$ & $-4.5(2.6)$ \\
Insulin treated & 71 & $13(0.7)$ & $10.2(2.5)$ & $-2.8(2.7)$ \\
Newly diagnosed & 24 & & $7.1(1.2)$ & $-6.4(1.6)$ \\
Non-insulin treated & 19 & $13.5(1.1)$ & $7.4(1.3)$ & $-5.9(1.5)$ \\
Insulin treated & 5 & $13.3(0.6)$ & & \\
Established diagnosis & 90 & $12.9(0.8)$ & $9.9(2.2)$ & $-3.1(2.3)$ \\
Non-insulin treated & 24 & $13(0.7)$ & $10.4(2.4)$ & $-2.6(2.6)$ \\
Insulin treated & 66 & & & \\
\hline
\end{tabular}

$A 1 C$ glycosylated hemoglobin

a A1C at month 12 or last observation carried forward

Table 5 Comparisons between newly and previously diagnosed subjects

\begin{tabular}{lccr}
\hline Parameter & $\begin{array}{l}\text { Newly diagnosed } \\
(\boldsymbol{n}=\mathbf{2 4})\end{array}$ & $\begin{array}{l}\text { Previously diagnosed } \\
(\boldsymbol{n}=\mathbf{9 0})\end{array}$ & $\boldsymbol{p}$ value \\
\hline Mean baseline A1C, \% (SD) & $13.4(1.0)$ & $-2.7(2.5)$ & 0.055 \\
Change in A1C from baseline ${ }^{\mathrm{a}}$, \% (SD) & $-6.3(1.5)$ & $37(41.1)$ & 0.001 \\
Responders , no. (\%) & $21(87.5)$ & $66(73.3)$ & $<0.001$ \\
Initial treatment with insulin, no. (\%) & $5(20.8)$ & $73(81.1)$ & $<(4.4 \%)$ \\
Insulin at some point, no. (\%) & $7(29.2)$ & $0.7(1.3)$ & $<0.001$ \\
Initial treatment with one non-insulin medication, no. (\%) & $1.2(1.5)$ & $139.2(84.1)$ & 0.13 \\
CDE visits, no. (SD) & $128.8(99.1)$ & 0.64 \\
First follow-up A1C, no. (SD) & &
\end{tabular}

$A 1 C$ glycosylated hemoglobin, $C D E$ certified diabetes educator

a Using last observation carried forward

b Achieved A1C $\leq 9 \%$ within 1 year

therapy. In addition, patients treated with insulin were actually less likely to achieve an $\mathrm{A} 1 \mathrm{C} \leq 9 \%$ and had less reduction in $\mathrm{A} 1 \mathrm{C}$ from baseline compared to non-insulin-treated patients. Possible explanations for this lack of effect with insulin include inadequate dose titration or sub-optimal adherence. Patients prescribed insulin could be more challenging to treat or more likely to have experienced past treatment failures.

This analysis did determine that more visits with a CDE was a predictor of successful 


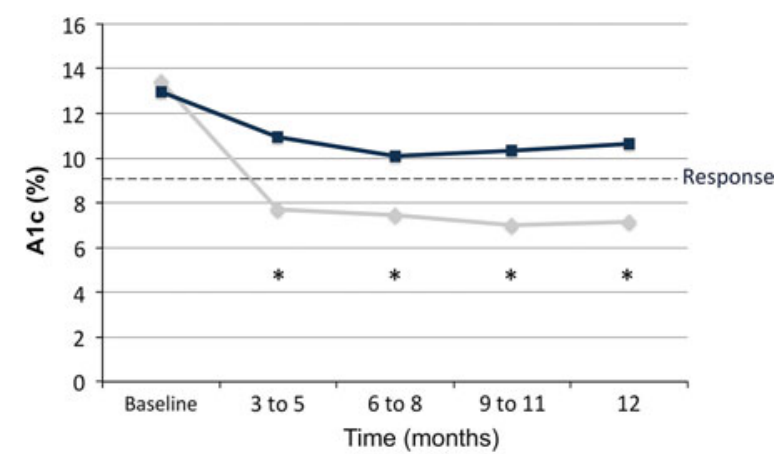

Fig. 1 Mean A1C levels over 12 months. Definitions: established diagnosis (squares), new diagnosis (diamonds). ${ }^{*} p$ value $<0.001$. AIC glycosylated hemoglobin

glycemic response. This finding is consistent with current clinical evidence and ADA and AACE consensus statements that emphasize diabetes self-management education as a vital component of diabetes care [6-8]. Responders also had fewer days to their first follow-up A1C compared to non-responders (120.0 vs. 154.7 days). While this difference suggests that close follow-up is an important component of glycemic control, our results show that even in this high-risk population, follow-up A1C monitoring is routinely delayed past the recommended 3 months.

A new diagnosis of diabetes was a confounding variable in our analysis. Patients who were newly diagnosed were much more likely to experience success than patients with an established diagnosis of T2D. Many newly diagnosed patients experienced success despite the fact that they were more likely to start a single non-insulin medication and less likely to use insulin at any point. This finding suggests that the time of diagnosis status (i.e., new versus established diagnosis) may be a key consideration when determining treatment approaches in addition to the severity of hyperglycemia. Prospective studies evaluating the safety and efficacy of non-insulin treatment options in newly diagnosed patients with severe hyperglycemia are warranted.

Several limitations to this study exist. First, this was a retrospective study performed at a single institutional system with a relatively small sample size and may not be able to be extrapolated to all patients with T2D and severe hyperglycemia. If more patients had been included, additional predictors of response might have been identified through this analysis. Second, selection bias is also a potential limitation of this study. The initial treatment regimen prescribed to a patient could have been impacted by patient factors not evaluated in the study. For example, patients that were prescribed insulin therapy could have been more difficult to treat or could have

Table 6 A1C achievement in newly diagnosed patients based on initial treatment

\begin{tabular}{lll}
\hline Initial diabetes treatment & $\begin{array}{l}\text { Achievement of } \\
\text { A1C } \mathbf{9 \% ,} \text { no. (\%) }\end{array}$ & $\begin{array}{l}\text { Achievement of } \\
\text { A1C } \mathbf{5 7 \% , ~ n o . ~ ( \% ) ~}\end{array}$ \\
\hline Total $(n=24)$ & $21(87.5)$ & $17(70.9)$ \\
One non-insulin medication $(n=16)$ & $14(58.3)$ & $10(41.7)$ \\
2 or more non-insulin medications $(n=3)$ & $3(12.5)$ & $3(12.5)$ \\
Insulin only regimen $(n=2)$ & $1(4.2)$ & $1(4.2)$ \\
Insulin + non-insulin regimen $(n=3)$ & $3(12.5)$ & $3(12.5)$ \\
\hline
\end{tabular}

$A 1 C$ glycosylated hemoglobin 
already failed non-insulin medications, making it more difficult to attain glycemic control. In addition, medication dose adjustments and adherence were not evaluated. This is an important limitation considering that other studies have identified that the most important factor in glycemic success with insulin is intensifying therapy $[9,10]$. It is highly likely that, in this realworld clinical setting, several patients in the insulin-treated cohort did not have their insulin regimen intensified sufficiently to achieve successful glycemic control. Third, we defined glycemic response as achieving an $\mathrm{A} 1 \mathrm{C} \leq 9 \%$ within the first year. This $\mathrm{A} 1 \mathrm{C}$ value does not meet the general target $\mathrm{A} 1 \mathrm{C}$ recommended by the ADA, but was determined by the authors, a priori, to constitute a clinically meaningful A1C reduction in this severe hyperglycemia population. Fourth, subjects were regarded as an "intent to treat" population. If a patient started therapy with a non-insulin medication within 1 month of baseline, they remained in the non-insulin cohort throughout analysis, regardless of whether they later received insulin medications.

\section{CONCLUSION}

The results from this retrospective study show that a new diagnosis of diabetes, more visits with a certified diabetes educator, and fewer days to first follow-up A1C were predictors of success in achieving an $\mathrm{A} 1 \mathrm{C} \leq 9 \%$, whereas insulin therapy was not. Furthermore, it was observed that insulin therapy did not lead to better A1C reductions or higher odds of achieving an A1C $\leq 9 \%$ compared to noninsulin regimens, regardless if the patient was newly diagnosed or had an established diagnosis. A new diagnosis of diabetes was a confounding variable. Meaningful glucose reductions were much more likely in newly diagnosed patients than those with established diabetes regardless of the treatment approach. Despite limitations, this study suggests that patients with T2D and severe hyperglycemia, particularly those newly diagnosed, may be able to be managed with non-insulin therapy. Further randomized prospective studies are warranted to confirm this.

\section{ACKNOWLEDGMENTS}

Grant support for NIH/NCRR Colorado CTSI grant number UL1 RR025780 provided access to the REDCap database. No funding was received for the publication of this article. Stefan $\mathrm{H}$. Sillau, University of Colorado, is acknowledged for providing statistical analysis support. Dr Trujillo is the guarantor for this article and takes responsibility for the integrity of the work as a whole.

Conflict of interest. William M. King IV, Michael T. McDermott, and Jennifer M. Trujillo all have no conflicts of interest to disclose.

Compliance with ethics guidelines. The analysis in this article is based on previously conducted studies and does not involve any new studies of human or animal subjects performed by any of the authors.

Open Access. This article is distributed under the terms of the Creative Commons Attribution Noncommercial License which permits any noncommercial use, distribution, and reproduction in any medium, provided the original author(s) and the source are credited. 


\section{REFERENCES}

1. American Diabetes Association. "Diabetes Statistics." http://www.diabetes.org/diabetes-basics/ diabetes-statistics/. Accessed 29 August 2011.

2. Chen HS, Wu TE, Jap TS, Hsiao LC, Lee SH, Lin HD. Beneficial effects of insulin on glycemic control and B-cell function in newly diagnosed type 2 diabetes with severe hyperglycemia after short-term intensive insulin therapy. Diabetes Care. 2008;31:1927-32.

3. Inzucchi SE, Bergenstal RM, Buse JB, et al. Management of hyperglycemia in type 2 diabetes: a patient-centered approach. Diabetes Care. 2012; 35:1364-79.

4. American Association of Clinical Endocrinologists. Statement by an American Association of Clinical Endocrinologists/American College of Endocrinology consensus panel on type 2 diabetes mellitus: an algorithm for glycemic control. Endocr Pract. 2009;15:540-59.

5. Harris PA, Taylor R, Thielke R, Payne J, Gonzalez N, Conde JG. Research electronic data capture (REDCap) - a metadata-driven methodology and workflow process for providing translational research informatics support. J Biomed Inform. 2009;42:377-81.

6. American Diabetes Association. Standards of Medical Care in Diabetes-2013. Diabetes Care. 2013;36(Suppl 1):S11-66.

7. Handelsman Y, Mechanick JI, Blonde L, et al. American Association of Clinical Endocrinologists Medical Guidelines for Clinical Practice for Developing a Diabetes Mellitus Comprehensive Care Plan. Endocr Pract. 2011;17(Suppl 2):1-53.

8. Stellefson M, Dipnarine K, Stopka C. The chronic care model and diabetes management in US primary care settings: a systematic review. Prev Chronic Dis. 2013;10:E26.

9. Nichols GA, Kimes TM, Harp JB, et al. Glycemic response and attainment of A1C goals following newly initiated insulin therapy for type 2 diabetes. Diabetes Care. 2012;35:495-7.

10. Nichols GA, Gandra SR, Chiou CF, et al. Successes and challenges of insulin therapy for type 2 diabetes in a managed-care setting. Curr Med Res Opin. 2010;26:9-15. 